\title{
Introduction: Eric Davidson and the molecular biology of evolution and development
}

\author{
Michel Morange ${ }^{1}$ - Ute Deichmann ${ }^{2}$
}

Published online: 13 October 2017

(C) Springer International Publishing AG 2017

\begin{abstract}
Between November 30th and December 2nd, 2015, the Jacques Loeb Centre for the History and Philosophy of the Life Sciences at Ben-Gurion University of the Negev in Beer Sheva (Israel) held its Eighth International Workshop under the title "From Genome to Gene: Causality, Synthesis and Evolution". Eric Davidson, the founder of the concept of developmental Gene Regulatory Networks, had regularly attended the previous meetings, and his participation in this one was expected, but he suddenly passed away 3 months before. In this paper, we provide an introduction and overview on five papers that were presented at the workshop and examine the importance of genomes and gene regulatory networks in extant biology, developmental biology, evolutionary biology and medicine, as well as a collection of remembrances of Eric Davidson, of his personality as well as of his scientific contributions. Historical perspectives are provided, and the ethical issues raised by the new tools developed to modify the genome are also discussed.
\end{abstract}

Keywords Developmental biology · Evolutionary biology · Gene regulatory network - Genome · Molecular medicine

Between November 30th and December 2nd, 2015, the Eighth International Workshop of the Jacques Loeb Centre for the History and Philosophy of the Life Sciences was held at Ben-Gurion University of the Negev in Beer Sheva (Israel). The workshop was entitled "From Genome to Gene: Causality, Synthesis and Evolution" and followed previous ones organized by Ute Deichmann at the Jacques

Ute Deichmann

uted@post.bgu.ac.il

1 Centre Cavaillès, Ecole Normale Supérieure, Paris, France

2 Beer Sheva, Israel 
Loeb Centre that had already explored the recent transformations of biology, their historical background and their scientific and philosophical impact. The eminent biologist Eric Davidson, who had been a regular participant and was expected to attend this workshop as well, passed away 3 months before it took place. This explains why a special session was organized during the meeting to honour his memory, and why these tributes are included in this issue. It might also explain why, spontaneously, many contributions were more or less focused on the relationships between genetics, developmental biology and evolution, the central issues that Eric Davidson addressed over his entire career. In the following we provide an introduction and overview to a topical collection of the journal History and Philosophy of the Life Sciences that we have put together from papers and remembrances presented at the workshop. Beyond personal recollections, the tributes also represent an introduction to the topics discussed in the papers by their respective authors.

The scientific work of Eric Davidson was emblematic not only of the molecular revolution-he was one of the founders of molecular embryology-but also of the transformations biology in general experienced with the importance attributed to genome sequencing, the rise of systems biology, and the progressive merging of evolutionary models and molecular explanations. Douglas Erwin reminds us in his obituary that Eric Davidson was one of the first biologists to link developmental and evolutionary mechanisms in the famous Britten-Davidson model of gene regulation published in 1969, long before the emergence of comparative evolutionarydevelopmental biology (Evo-Devo) in the 1980s. Eric Davidson was also one of the few molecular biologists and geneticists to incorporate observations originating in the study of fossils into his reflections and models. The importance of the Gene Regulatory Network (GRN) model of sea urchin development that he built at the end of his career was not immediately acknowledged by all biologists, but it definitely was one of the most ambitious and fruitful models ever proposed to explain not only early development but also evolutionary transformations at the molecular level.

Ute Deichmann reminds us in her recollections that Eric Davidson had a deep interest in historical and philosophical studies of biology. As he dared to state, in his characteristic style, he had the ambition not only to be a brilliant scientist, but also an intellectual, an ambition unfortunately not shared by many biologists today. He critically commented on tendencies that he considered detrimental to researchholistic, anti-mechanistic scientific views as well as the narrow-mindedness he perceived in the attitudes of his fellow molecular biologists who had not realized the importance of systems approaches for the explanation of complex phenomena such as development. He was also critical of neo-Darwinism for its failure to explain the origin of major evolutionary novelties and innovations.

Michel Morange reveals and explains from his first-hand experience the tensions between the French school of molecular genetics, in particular François Jacob and Jacques Monod, and Eric's work concerning the explanatory scope of their respective models of gene regulation. He also reflects on Eric's personal characteristics such as his enthusiasm and his ability to engage, until the end of his life, in strong intellectual relationships with people from very different 
backgrounds. These personal characteristics help explain Eric's deep commitment to the solution of problems he perceived as fundamental and the breadth of his scope. His characteristics as a challenging teacher are finally portrayed in the contribution by Smadar Ben-Tabou de-Leon.

These four memorial essays are followed by essays that go back to papers presented at the workshop and explore recent transformations of biology from different perspectives. Ute Deichmann uses a broad historical and philosophical perspective to show that the beginning of biology as a modern experimental science was closely related to the introduction of the concepts of hierarchical organization and biological specificity (later genetic information) in basic areas of research. She also demonstrates that causal explanations of development and evolution progressively centred around the gene and genome during most of the twentieth century. She goes on to describe late twentieth century tendencies in philosophy and biology that call into question the privileged role of the genome in the causation of development and argue for the replacement of reductionist, gene-centred explanations with holistic ones that focus on environmental influences. Contrasting this view, Deichmann argues that there are more fertile replacements for molecular 'reductionism', namely a synthesis of genomics, embryology, biochemistry, and computer science that results in research that is as exact and causally predictive as earlier molecular biology and yet more efficient in explaining development and evolution. Here the concepts of hierarchy (e.g. of Gene Regulatory Networks), genomic causality and specificity maintain their central place.

The idea of simple causal explanations has also been challenged in research on genetic diseases as is shown in the contribution by Shannon Keenan and Stanislav Shvartsman. They make clear that despite major advances in research on genetic diseases, major conceptual and technical challenges have remained, which they illustrate by discussing the causal links between molecular mechanisms and systems-level phenotypes in molecular diseases. They contrast the rather simple case of sickle cell anemia with the complex case of RASopathies where the effects of the mutated gene are not limited to one cell type, but occur within a complex network of interacting proteins.

The idea of the causal role of genes has generated the hope of curing genetic diseases and transforming human characteristics by modifying the genome. Michel Morange reminds us that these ambitions have anticipated a precise knowledge of the chemical nature of the genes, as well as the existence of tools permitting manipulation of the genome. A new generation of molecular tools has recently emerged that permit the "editing of the genome". CRISPR-Cas9 is the most famous of these new tools, the result of a complex and tortuous history. These new tools will be highly useful to biologists. It is not obvious, however, that they will support the ambitious projects described previously. Other strategies have been developed to prevent the occurrence of genetic diseases. Michel Morange concludes that the biological transformation of human species is no longer seen as a priority, but rather as an additional risk for humankind.

Douglas Erwin reconsiders issues that were discussed by evolutionary biologists for more than a century, since Darwin and even before: the relative roles of what later came to be called mutation and selection in the occurrence of evolutionary 
innovations and the existence (or lack thereof) of macroevolutionary mechanisms besides microevolutionary ones. Since the Modern Synthesis, most evolutionary biologists have maintained that microevolutionary processes carried on over sufficient time will generate macroevolutionary patterns, with no need for other pattern-generating mechanisms. Stephen Jay Gould challenged this emphasis on selection and microevolution already in the 1970s. Erwin shows that, more recently, comparative studies of developmental evolution have pointed to the origin of variants as a driving macroevolutionary force in contrast to most macroevolutionary studies that have focused on the distribution of macroevolutionary patterns, not the origin of variation. He mentions particularly the repatterning of gene regulatory networks as a possible mechanism for generating developmental novelties.

The distinction between phenotypic novelty and innovation (the latter reflecting ecological and evolutionary success) and the fact that long macroevolutionary lags between the generation of novel phenotypes and their evolutionary success were discovered in many clades make Erwin believe that ecological opportunity may actually be of little importance. Though the question raised in his title, whether evolution is due to a developmental push or an environmental pull remains to be finally answered, Erwin provides a wonderfully clear and comprehensive description of the recent approaches to this issue by developmental biologists, evolutionary biologists and paleontologists, and shows how in recent decades they have tried to articulate the two opposing conceptions of phenotypic novelty and innovation.

The contribution of Ellen Rothenberg comprises the best conclusion to the series of articles presented in the topical collection. She considers what will remain the main contribution of Eric Davidson-the construction of the sea urchin developmental GRN and the demonstration, through modelling, of its capacity to account for the early development of this organism. She argues that this model, adapted to the early development of sea urchins, cannot be directly transferred to stem-cell based development, in particular to the development of the haematopoietic and immune systems in vertebrates. New phenomena have to be included in the model: the existence of a subtle dosage effect for transcription factors, and the (epigenetic) effect of cellular regulatory history. These properties fit the functions that mammalian hematopoiesis has evolved to carry out as a system in which-unlike in early development-a great variety of cell types is generated. Her contribution shows that models, whatever their demonstrated explanatory power, must remain open to transformations, as genes and their products are constantly adapted to new environments and functions in the course of evolution. Her paper is both an homage to the pioneering work of Eric Davidson, and a reminder that challenging the present state of knowledge and opening up new perspectives in which models may have to fulfil very different needs is an ongoing necessity for keeping scientific models fruitful and preventing them from being converted into dogmas. 